\title{
ANÁLISIS ESTADÍSTICO DEL ESPESOR DE RECUBRIMIENTO DEL ALUMINIO LACADO EN EL ENSAYO DE NIEBLA SALINA NEUTRA
}

\author{
Statistical Analysis of the Coating Thickness \\ of the Lacquered Aluminium in the \\ Neutral Saline Fog Test
}

\begin{abstract}
Jeyris Martínez Gutiérrez ${ }^{1}$, Rigoberto Marrero Águila², Emilio M. Lluveras Pérez ${ }^{3}$, Rosabel López Pino ${ }^{4}$
\end{abstract}

\author{
${ }^{1,3}$ Laboratorio de Ensayos de Tropicalización-LABET, La Habana, Cuba \\ 2,4 Universidad Tecnológica de La Habana José Antonio Echeverría, La Habana, Cuba.
}

\begin{abstract}
RESUMEN
La belleza natural del aluminio, la combinación con una gran variedad de formas y colores que se pueden alcanzar, la alta resistencia a la corrosión y sus magnificas propiedades estructurales, han hecho de este material un metal ideal para la construcción. La tendencia actual en cuanto a los cambios climáticos hace que la atmósfera se torne cada vez más agresiva, por lo que surgen inquietudes acerca de la degradación del recubrimiento en determinadas condiciones. Teniendo en cuenta que los estudios referidos al tema necesitan tiempos excesivamente largos de exposición, se toma la variante de la utilización de ensayos climáticos acelerados. El presente trabajo tiene como objetivo realizar una comparación de las medias y desviaciones de las distribuciones del espesor del aluminio lacado, antes y después del ensayo de niebla salina neutra en condiciones extremas de humedad, temperatura y salinidad. Se demostró la influencia de la acción de la niebla salina neutra sobre el espesor a partir de las diferentes pruebas realizadas en el software Statgraphics Centurion, evidenciándose un cambio en las distribuciones normales en los valores obtenidos antes $y$ después de ser realizado el ensayo. Se observa una tendencia a la disminución del espesor y la existencia de diferencias significativas en las medias y desviaciones estándares con un $95 \%$ de confiabilidad, pero aun asi los resultados de los ensayos resultaron conformes teniendo en cuenta el cumplimiento de las directrices de Qualicoat.
\end{abstract}

Palabras clave: aluminio lacado, espesor, estadistica, resistencia.

\footnotetext{
${ }^{1}$ jeyris@labet.ctec.cu, orcid.org/0000-0002-0564-8661

${ }^{2}$ rigo@quimica.cujae.edu.cu, orcid.org/0000-0002-3011-5701

${ }^{3}$ emilio@labet.ctec.cu, orcid.org/0000-0002-3648-6204

${ }^{4}$ rosabelequimica.cujae.edu.cu, orcid.org/0000-0002-0535-3599
} 


\section{Abstract}

The natural beauty of aluminum, the combination with a variety of shapes and colors that can be achieved, the high resistance to corrosion and its magnificent structural properties, have made this material an ideal metal for construction. The current trend in terms of climate changes makes the atmosphere increasingly aggressive, so that concerns arise about the degradation of the coating under certain conditions. Taking into account that the studies referred to the subject need excessively long exposure times, the variant of the use of accelerated climatic tests is taken. The objective of this work is to make a comparison of the means and deviations of the thickness distributions of lacquered aluminum, before and after the neutral salt spray test under extreme conditions of humidity, temperature and salinity. The influence of the action of the neutral salt spray on the thickness was demonstrated from the different tests carried out in the Statgraphics Centurionsoftware, showing a change in the normal distributions in the values obtained before and after the test was carried out. There is a tendency to decrease the thickness and the existence of significant differences in standard means and deviations with a $95 \%$ reliability, but even so the results of the tests were found taking into account the compliance with the Qualicoat guidelines.

Key words: lacquered aluminum, resistance, statistics, thickness.

\section{INTRODUCCIÓN}

"El desarrollo histórico de la evolución industrial de los materiales para fabricar elementos estructurales o en instalaciones industriales ha buscado mejorar sus propiedades a través de los adelantos tecnológicos que se han ido presentando. Una alternativa económica y competitiva ha sido la aplicación de recubrimientos orgánicos, los cuales son empleados ampliamente en el sector industrial, porque incrementan la resistencia a la corrosión de los materiales metálicos que se encuentran sometidos a ambientes agresivos" (Olaya et al., 2012, 45).

La belleza natural del aluminio, la combinación con una gran variedad de formas y colores que se pueden alcanzar, la alta resistencia a la corrosión y sus magníficas propiedades estructurales, han hecho de este material un metal ideal para la construcción, en general, satisfaciendo las más altas exigencias de ingenieros y arquitectos. Con el objetivo de proteger al aluminio y sus aleaciones frente a la corrosión se suele recurrir al lacado (barrera entre el metal y el medio corrosivo) como uno de los medios de protección (González, 2013, 19).
La corrosión puede ser definida como una reacción irreversible de un material con el medio ambiente, lo que resulta en la degradación del material y la pérdida de sus propiedades (Cabrera, 2016, 7).

El buen comportamiento a la corrosión del aluminio es debido a la presencia permanente sobre el metal de una capa de óxido natural constituido por óxido de aluminio (alúmina), que le hace pasivo a la acción del medio ambiente. Aunque de muy pequeńo espesor (entre 5 y $10 \mathrm{~nm}$ ), se forma instantáneamente desde que el metal entra en contacto con un medio oxidante, y su presencia tiene una gran importancia en la resistencia a la corrosión del aluminio. La formación y disolución de esta capa de óxido depende de varios factores, fundamentalmente de los elementos constituyentes de aleación, la humedad y salinidad del ambiente, del pH y de la temperatura (Foncubierta, 2017, 11).

En la actualidad son muchos los productores de carpintería de aluminio lacado en el mundo, y en Cuba, son cada vez más las obras en las que se observa la instalación de puertas y ventanas de aluminio lacado (González, 2013, 2). 
Cuba es un archipiélago con un clima caracterizado por tener más de la mitad del año temperaturas medias, aproximadamente de $25{ }^{\circ} \mathrm{C}$, y humedad relativa media alrededor del $75 \%$, respectivamente. Dada su configuración y ubicación geográfica, la influencia del aerosol marino alcanza casi todo el territorio nacional (Castańeda, 2014, 53).

La tendencia actual en cuanto a los cambios climáticos hace que la atmósfera se torne cada vez más agresiva, por lo que surgen inquietudes acerca de la degradación del recubrimiento en determinadas condiciones.

Teniendo en cuenta que los estudios referidos al tema necesitan tiempos excesivamente largos de exposición, se toma la variante de la utilización de ensayos climáticos acelerados. La cámara de ensayo de niebla salina neutra permite someter las muestras a la acción de una niebla de sal atomizada en condiciones prefijadas de presión, concentración y temperatura. Existen regulaciones referidas a la evaluación de la calidad de estos recubrimientos y se han establecido directrices que tienen que ser cumplidas por los productores para ser reconocidos y poder optar por su sello de calidad.

La estadística experimental -en el mundo moderno- es una necesidad real, presente en todas las áreas del conocimiento humano, como una herramienta para auxiliar las decisiones a ser tomadas, permitiendo mayor seguridad a la investigación a ser transformada en tecnología y utilizada por la humanidad o por parte de ella (Lluveras et al., 2018, 179).

El presente trabajo tiene como objetivo determinar si existen diferencias estadísticas significativas en las distribuciones del espesor del aluminio lacado, antes y después de la influencia de la acción producida por la cámara de niebla salina neutra. La comparación de las desviaciones y las medias serán realizadas en el software Statgraphics Centurion.

\section{Materiales y MÉTOdos}

Con el objetivo de evaluar el comportamiento del espesor de recubrimiento del aluminio lacado, ante la influencia de factores climáticos simulados en el ensayo climático acelerado de niebla salina neutra, se realiza una comparación de las distribuciones de antes y después del ensayo: las probetas se expusieron durante un tiempo de 1.000 h. Para mantener los derechos de confidencialidad se adoptó una identificación general de la muestra utilizada. A continuación, se describen los procedimientos realizados para cada ensayo.

\subsection{Procedimiento}

Antes de su utilización cada instrumento debe ser calibrado conforme a las indicaciones del fabricante y usando los patrones de espesor apropiados. Durante la utilización el calibrado debe ser verificado a intervalos frecuentes.

Se elimina previamente de la superficie a ensayar todas las partículas extrañas, tales como suciedades, aceites, grasas y productos de la corrosión, sin eliminar nada de material del recubrimiento.

Se coloca el sensor sobre la capa de revestimiento a medir. La lectura en la pantalla es el espesor de la capa de revestimiento.

Para tomar la próxima medición simplemente se levanta el sensor a más de $1 \mathrm{~cm}$ y se repite el paso anterior (NC ISO 2808, 2010, 26-32)

\subsection{Ensayo de niebla salina neutra}

La cámara de ensayo modelo Q-FOG utilizada en este estudio presenta una capacidad de $1,1 \mathrm{~m}^{3}$ la cual permite someter las probetas de aluminio lacado a condiciones severas de temperatura, humedad y salinidad, permitiéndose la valoración de la resistencia a la corrosión de este material con materiales metálicos con o sin protección de la corrosión permanente o temporal como se muestra en la Figura 1. 


\subsubsection{Procedimiento}

La preparación de la solución de cloruro de sodio se realiza disolviendo una cantidad suficiente de cloruro de sodio en agua destilada o desionizada con una conductividad no mayor que $20 \mu \mathrm{S} / \mathrm{cm}$ a $25^{\circ} \mathrm{C}$ para obtener una concentración de $50 \pm 5 \mathrm{~g} / \mathrm{L}$ (NC ISO 9227, 2014, 6-26).

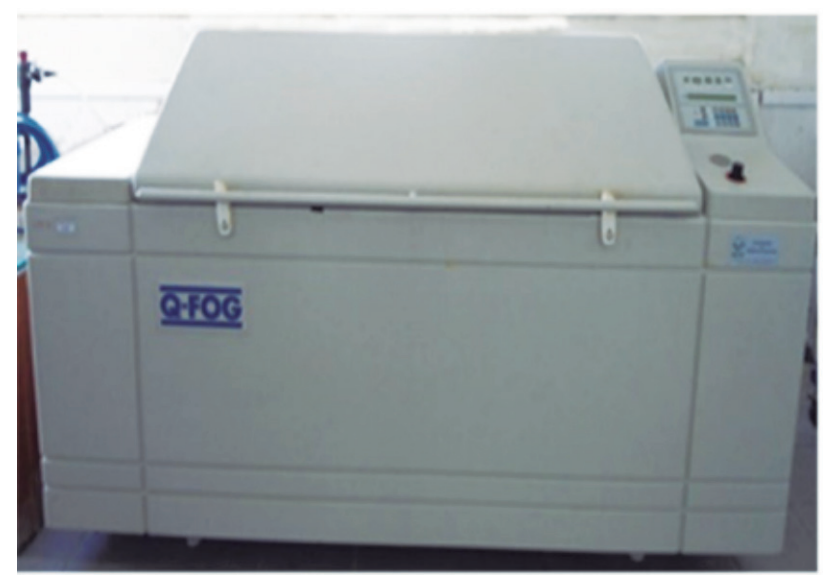

Fig. 1 Cámara de ensayo de niebla salina neutra Modelo Q- FOG.

La concentración de cloruro de sodio de la solución colectada del rociado debe ser $50 \mathrm{~g} / \mathrm{L} \pm 5 \mathrm{~g} / \mathrm{L}$. El intervalo de gravedad específica para una solución de 50 $\pm 5 \mathrm{~g} / \mathrm{L}$ es de 1,029 a $1,032 \mathrm{~g} / \mathrm{cm}^{3}$ a $25^{\circ} \mathrm{C}$. El cloruro de sodio debe contener no menos de 0,001 \% masa de cobre y menos de $0,001 \%$ masa de níquel determinado por espectrofotometría de absorción atómica u otro método analítico de similar sensibilidad. El mismo no debe contener más de $0,1 \%$ masa de yoduro de sodio o más de $0,5 \%$ masa de impurezas totales calculadas para la sal seca.

Si es necesario se filtra la solución antes de colocarla en el recipiente del aparato para eliminar cualquier materia sólida que pueda bloquear las aberturas del dispositivo de rociado.

Las probetas se ubicaron en portamuestras resistentes a la corrosión por la solución de rociado para descartar la influencia de su corrosión en las condiciones de ensayo. Se exponen un período de $1.000 \mathrm{~h}$.
Al finalizar el período de ensayo, se sacan las probetas de la cabina y se dejan secar por $0,5 \mathrm{~h}$ a $1 \mathrm{~h}$ antes de enjuagar, para reducir el riesgo de eliminar los productos de corrosión. Después se examinan, removiendo cuidadosamente los residuos de la solución de rociado de sus superficies. Las probetas se enjuagan con agua corriente limpia a una temperatura de $23 \pm 2$ ${ }^{\circ} \mathrm{C}$. Fundamentalmente se determina la resistencia a la formación de ampollas, la presencia de oxidación, grietas, decoloración, descamación, enyesado, pérdidas de brillo en las películas de pinturas (NC ISO 4628 1-6, 2007, 1-18).

\subsection{Ensayo de determinación de los espesores de recubrimientos sobre sustratos no magnéticos}

Se emplea el método no destructivo para la determinación del espesor de recubrimiento, no conductores sobre materiales base no magnéticos eléctricamente conductores (UNE EN ISO 2360, 2004, 1-10). Las mediciones se realizan con un medidor de espesores de recubrimientos que incorpora los principios de medida de corrientes de Foucault, como se muestra en la Figura 2.

El espesor del revestimiento de cada pieza a controlar se deberá medir sobre la superficie significativa, $\mathrm{y}$ al menos en cinco puntos de medida (alrededor de 1 $\mathrm{cm}^{2}$ ), procediéndose a 5 lecturas individuales del espesor en cada punto.

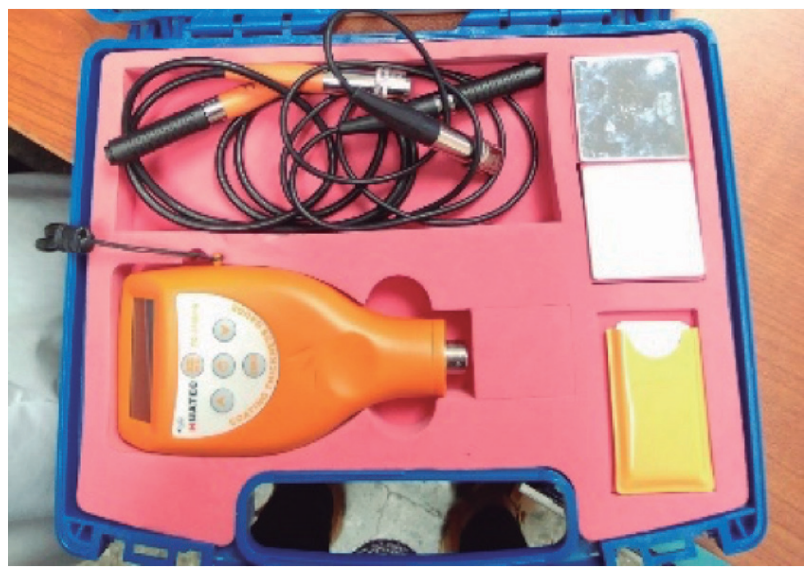

Fig. 2 Medidor de espesor del recubrimiento utilizado para el ensayo. HUATEC TG-2100FN 


\section{AnÁlisis estadístico}

El análisis estadístico de este trabajo se basa en la realización de una comparación entre las medias y desviaciones estándares, teniendo en cuenta los valores obtenidos de espesor de recubrimiento de tres probetas, antes y después de ser sometidas al ensayo climático acelerado de niebla salina neutra.

En cada probeta se realizó una medición y cuatro réplicas obteniéndose en total 15 mediciones.

Se emplea el Statgraphics Centurion XVII con el objetivo de aumentar la fiabilidad de los resultados obtenidos.

\subsection{Procedimiento}

Se introducen los valores obtenidos de espesor en las columnas correspondientes y se selecciona la opción: Comparar/ Dos muestras/ Muestras independientes. En este caso el software Statgraphics define las muestras como las distribuciones de los valores del muestreo antes y después del ensayo acelerado.

En la ventana tablas y gráficos, se debe seleccionar las opciones siguientes: resumen del análisis, resumen estadístico, comparación de medias, comparación de desviaciones estándar, histograma y gráfico de caja y bigotes.

\section{RESULTAdos y ANÁLISIS}

\subsection{Resistencia a la niebla salina}

Se colocaron las tres probetas de aluminio lacado en la cámara de niebla salina neutra durante un tiempo de exposición de 1.000 h, las mismas fueron marcadas por una incisión de $1 \mathrm{~mm}$ de ancho con un instrumento cortante, dejando al descubierto el metal para verificar el avance de la corrosión en sus bordes, como se evidencia en la Figura 3.
Las probetas fueron evaluadas durante el ensayo y al ser extraídas no se observó la formación de ampollas, la presencia de oxidación, grietas, decoloración, descamación, enyesado ni pérdidas de brillo en la película de pintura, según lo establecido en la norma de evaluación de la degradación de los recubrimientos de pintura (NC ISO 4628, 2007, 1-6). El avance de la corrosión al nivel de la incisión no excede de $1 \mathrm{~mm}$, cumpliéndose con las exigencias establecidas en las directrices de Qualicoat.
A

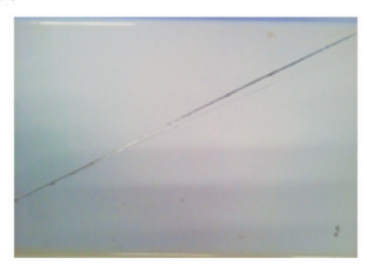

B

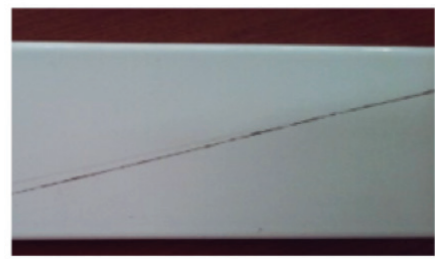

Fig. 3 Imagen obtenida de una probeta ensayada en la cámara de niebla salina neutra.

A: antes de comenzar el ensayo

B: después de finalizado el ensayo

\subsection{Determinación de los espesores de recubri- mientos sobre sustratos no magnéticos}

Antes de ubicar las probetas a ensayar en la cámara de niebla salina neutra, se determinaron según el procedimiento, los valores mínimos, máximos y promedio del espesor del recubrimiento lacado. En las tablas 1 y 2 se exponen los resultados obtenidos antes y después de los ensayos climáticos acelerados.

\subsection{Análisis estadístico}

A continuación, en las Figuras 4 y 5 y la Tabla 3, se observan los datos analizados para el espesor del recubrimiento de las probetas ensayadas. 
- Análisis estadístico del espesor de recubrimiento del aluminio lacado en el ensayo de niebla salina neutra

Jeyris Martínez Gutiérrez, Rigoberto Marrero Águila, Emilio M. Lluveras Pérez, Rosabel López Pino

\section{Tabla 1.}

Resultados obtenidos en las determinaciones de espesor en las probetas ensayadas antes de ser sometidas a la niebla salina neutra

\begin{tabular}{|c|c|c|c|c|c|c|c|c|c|}
\hline \multirow{2}{*}{ PROBETAS } & \multicolumn{5}{|c|}{ MEDICIONES } & \multirow{2}{*}{$\begin{array}{l}\text { VALOR } \\
\text { MÍNIMO } \\
(\mu \mathrm{M})\end{array}$} & \multirow{2}{*}{$\begin{array}{c}\text { VALOR } \\
\text { MÁXIMO } \\
(\mu \mathrm{M})\end{array}$} & \multirow{2}{*}{$\begin{array}{l}\text { PROMEDIO } \\
(\mu \mathrm{M})\end{array}$} & \multirow{2}{*}{$\begin{array}{c}\text { INCERTIDUMBRE } \\
\text { UC }(\mu \mathrm{M})\end{array}$} \\
\hline & 1 & 2 & 3 & 4 & 5 & & & & \\
\hline 1 & 92,0 & 91,5 & 94,5 & 95,3 & 93,4 & 91,5 & 95,3 & 93,3 & 0,0111 \\
\hline 2 & 91,1 & 94,0 & 94,7 & 92,2 & 92,8 & 91,1 & 94,7 & 92,9 & 0,0111 \\
\hline 3 & 94,7 & 93,4 & 93,8 & 95,6 & 94,4 & 93,4 & 95,6 & 94,3 & 0,0111 \\
\hline
\end{tabular}

Fuente: autores

TABLA 2.

Resultados obtenidos en las determinaciones de espesor en las probetas ensayadas después de ser sometidas a la niebla salina neutra

\begin{tabular}{|c|c|c|c|c|c|c|c|c|c|}
\hline \multirow{2}{*}{ PROBETAS } & \multicolumn{5}{|c|}{ MEDICIONES } & \multirow{2}{*}{$\begin{array}{c}\text { VALOR } \\
\text { MÍNIMO } \\
(\mu \mathrm{M})\end{array}$} & \multirow{2}{*}{$\begin{array}{c}\text { VALOR } \\
\text { MÁXIMO } \\
(\mu \mathrm{M})\end{array}$} & \multirow{2}{*}{$\begin{array}{l}\text { PROMEDIO } \\
(\mu \mathrm{M})\end{array}$} & \multirow{2}{*}{$\begin{array}{l}\text { INCERTIDUMBRE } \\
\text { UC }(\mu \mathrm{M})\end{array}$} \\
\hline & 1 & 2 & 3 & 4 & 5 & & & & \\
\hline 1 & 81,3 & 82,5 & 84,3 & 84,3 & 83,1 & 81,3 & 84,3 & 83,1 & 0,0111 \\
\hline 2 & 79,0 & 78,0 & 77,0 & 78,5 & 79,0 & 77,0 & 79,0 & 78,3 & 0,0111 \\
\hline 3 & 71,8 & 72,9 & 74,4 & 72,3 & 70,7 & 70,7 & 74,4 & 72,4 & 0,0111 \\
\hline
\end{tabular}

Fuente: autores

Resultados obtenidos en las determinaciones de espesor en las probetas ensayadas después de ser sometidas a la niebla salina neutra
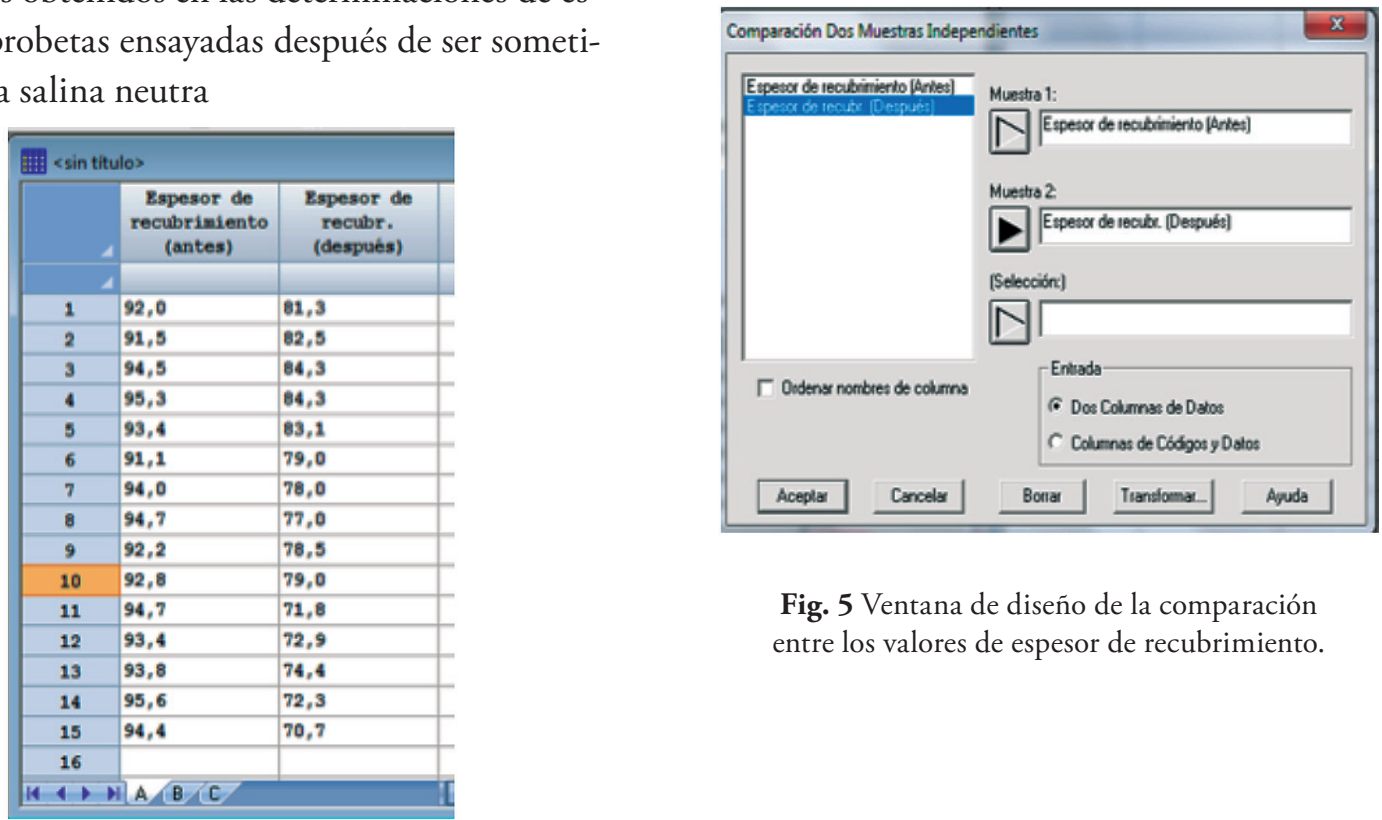

Fig. 5 Ventana de diseño de la comparación entre los valores de espesor de recubrimiento.

Fig. 4 Datos introducidos en el Statgraphics enturion XVII.

Fuente: autores 
TABLA 3.

Resumen estadístico

\begin{tabular}{|c|c|c|}
\hline & $\begin{array}{c}\text { ESPESOR } \\
\text { (ANTES) }\end{array}$ & $\begin{array}{c}\text { ESPESOR } \\
\text { (DESPUÉS) }\end{array}$ \\
\hline Recuento & 15 & 15 \\
\hline Promedio & 93,56 & 77,94 \\
\hline $\begin{array}{c}\text { Desviación } \\
\text { estándar }\end{array}$ & 1,38 & 4,65 \\
\hline $\begin{array}{c}\text { Coeficiente } \\
\text { de variación }\end{array}$ & $1,48 \%$ & $5,97 \%$ \\
\hline Mínimo & 91,1 & 70,7 \\
\hline Máximo & 95,6 & 84,3 \\
\hline Rango & 4,5 & 13,6 \\
\hline $\begin{array}{c}\text { Sesgo } \\
\text { estandarizado }\end{array}$ & $-0,60$ & $-0,23$ \\
\hline $\begin{array}{c}\text { Curtosis } \\
\text { estandarizada }\end{array}$ & $-0,72$ & $-1,03$ \\
\hline
\end{tabular}

Fuente: los autores

En este caso, ambos valores de sesgo estandarizado y la curtosis se encuentran dentro del intervalo esperado. Ambas muestras tienen valores de asimetría y curtosis estandarizadas en el intervalo entre -2 y 2. Los valores en dicho intervalo son consistentes con la hipótesis de que los datos provienen de distribuciones normales.

La desviación típica de los valores de espesor del recubrimiento antes de ser ensayadas las probetas es menor que la de los valores obtenidos al finalizar el ensayo.

\subsubsection{Histograma dual}

El histograma dual de frecuencias compara las dos distribuciones simultáneamente, como se muestra en la Figura 6. El histograma para los valores de espesor antes de ser sometidas las probetas al ensayo de niebla salina, se encuentra por encima de la línea horizontal, con un desplazamiento hacia la derecha. Las formas de las distribuciones son similares.

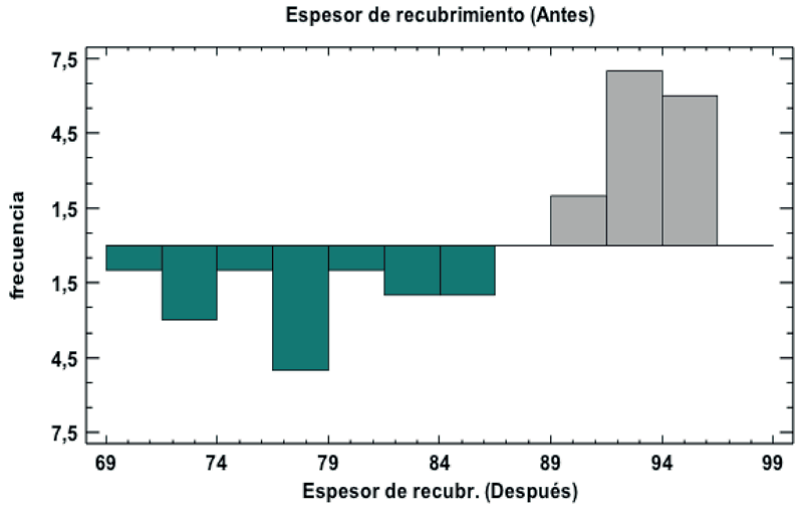

Fig. 6 Histograma de frecuencias dual

\subsubsection{Gráfico dual de caja y bigotes}

La ventana de análisis se muestra en la Figura 7, siendo un gráfico de caja y bigotes para los valores de espesor obtenidos antes y después del ensayo. La caja central cubre la mitad de las observaciones de cada distribución. Los bigotes se extienden entre los valores máximo y mínimo de cada muestra, excepto para algunos puntos que se sitúan inusualmente lejanos a las cajas. Se dibuja una línea vertical en cada caja a la altura de la mediana, mientras que los pequeńos signos más situados en el interior de las cajas indican las localizaciones de las medias muestrales.

Se evidencia un aparente desvío del centro de la distribución hacia la izquierda. Las medias y medianas muestran una diferencia similar. El intervalo cubierto por los valores de espesor obtenidos después del ensayo es más ancho que el intervalo cubierto por los valores de espesor obtenidos antes del ensayo. 


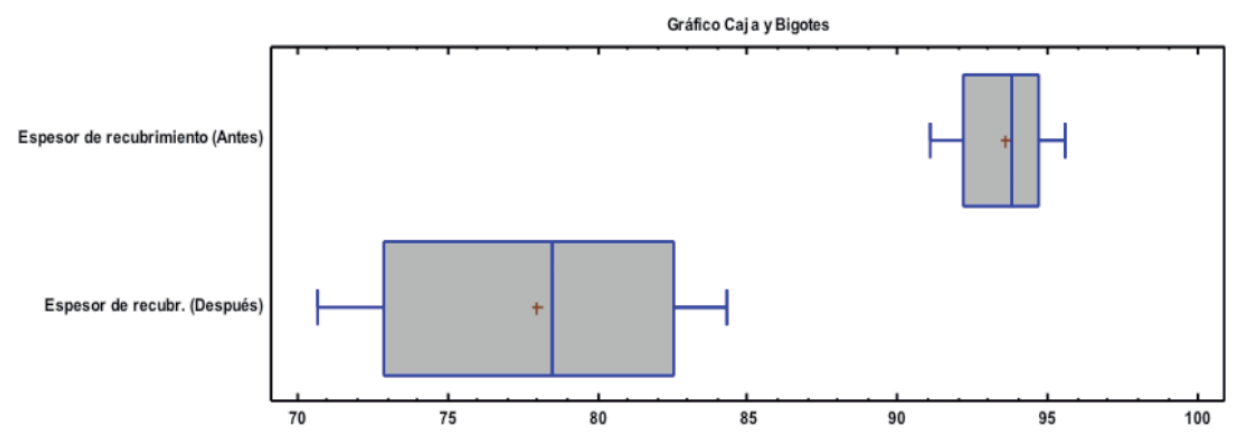

Fig. 7 Gráfico dual de caja y bigotes

\subsubsection{Comparación de medias}

A continuación, se muestran los resultados obtenidos en la prueba $t$, donde se realiza una comparación de las medias, los intervalos de confianza y la diferencia entre las mismas.

- Intervalos de confianza del 95,0 \% para la media de espesor de recubrimiento (antes): 93,56+/- 0,76 [92,79; 94,32]

- Intervalos de confianza del 95,0 \% para la media de espesor de recubrimiento. (después): 77,94 +/- 2,57 $[75,36 ; 80,51]$

- Intervalos de confianza del 95,0 \% intervalo de confianza para la diferencia de medias suponiendo varianzas iguales: $15,62+/-2,56[13,05 ; 18,18]$

Prueba t para comparar las medias:

Hipótesis nula: media $_{1}=$ media $_{2}$

Hipótesis alternativa: media $_{1} \neq$ media $_{2}$

Suponiendo varianzas iguales: $\mathrm{t}=12,4651$ valor $-\mathrm{P}=0$. Se rechaza la hipótesis nula para alfa $=0,05$. De interés particular es el intervalo de confianza para la diferencia entre las medias, el cual se extiende desde 13,05 hasta 18,18 . Puesto que el intervalo no contiene el valor 0 , existe una diferencia estadísticamente significativa entre las medias de las dos distribuciones, con un nivel de confianza del 95,0\%.

También se utiliza la prueba t para la evaluación de las hipótesis específicas acerca de la diferencia entre las medias de las poblaciones, de las cuales provienen las dos distribuciones. En este caso, la prueba se ha construido para determinar si la diferencia entre las dos medias es igual a 0,0 versus la hipótesis alterna de que la diferencia no es igual a 0,0 . Puesto que el valor-P calculado es menor que 0,05 , se puede rechazar la hipótesis nula en favor de la alterna.

\subsubsection{Comparación de desviaciones estándares}

A continuación, se muestran los resultados obtenidos en la prueba $F$ para comparar las varianzas de las dos muestras, intervalos de confianza y la razón entre las mismas. Ver Tabla 4.

Razón de varianzas= 0,0885922, intervalos de confianza del 95,0\%

- Desviación estándar de recubrimiento (antes):

$[1,01 ; 2,18]$

- Desviación estándar de recubrimiento (después): $[3,40 ; 7,33]$

- Razones de varianzas: $[0,02 ; 0,26]$

Prueba F para comparar desviaciones estándares: Hipótesis nula: $\operatorname{sigma}_{1}=\operatorname{sigma}_{2}$ Hipótesis alternativa: $\operatorname{sigma}_{1} \neq \operatorname{sigma}_{2}$

$\mathrm{F}=0,0885922$ valor- $\mathrm{P}=0,0000521656$, se rechaza la hipótesis nula para alfa $=0,05$. 
TABLA 4.

Resultados obtenidos en la prueba-F

\begin{tabular}{|c|c|c|}
\hline & $\begin{array}{c}\text { ESPESOR } \\
\text { (ANTES) }\end{array}$ & $\begin{array}{c}\text { ESPESOR } \\
\text { (DESPUÉS) }\end{array}$ \\
\hline $\begin{array}{c}\text { DESVIACIÓN } \\
\text { ESTÁNDAR }\end{array}$ & 1,38 & 4,65 \\
\hline VARIANZA & 1,92 & 21,64 \\
\hline $\begin{array}{c}\text { GRADOS DE } \\
\text { LIBERTAD }\end{array}$ & 14 & 14 \\
\hline
\end{tabular}

De particular interés es el intervalo de confianza para la razón de varianzas, el cual se extiende desde 0,02 hasta 0,26 . Puesto que el intervalo no contiene el valor de 1 , existe diferencia estadísticamente significativa entre las desviaciones estándar de las dos muestras con un $95,0 \%$.

También puede ejecutarse una prueba $\mathrm{F}$, para evaluar una hipótesis específica acerca de las desviaciones estándares de las poblaciones de las cuales provienen las dos distribuciones. En este caso, la prueba se ha construido para determinar si el cociente de las desviaciones estándares es igual a 1,0 versus la hipótesis alternativa de que el cociente no es igual a 1,0 . Puesto que el valor-P calculado es menor que 0,05 , se puede rechazar la hipótesis nula en favor de la alterna.

\section{Conclusiones}

Se demostró la influencia de la acción de la niebla salina neutra sobre el espesor de recubrimiento lacado, a partir de las diferentes pruebas realizadas con el software Statgraphics Centurion XVII, evidenciándose la distribución normal en los valores obtenidos antes y después de ser realizado el ensayo, con una tendencia a la disminución y la existencia de diferencias significativas en las medias y distribuciones estándares con un $95 \%$ de confiabilidad.

Se pudo evidenciar la resistencia del recubrimiento de pintura sobre el aluminio ante las condiciones extremas simuladas de humedad, temperatura y salinidad, a pesar de variar en las medias y distribuciones de antes y después.

Se evidencia el cumplimiento de las directrices de Qualicoat para el aluminio lacado, puesto que se cumple con las exigencias establecidas, no se observa la aparición de defectos que denoten la degradación del recubrimiento durante el ensayo de niebla salina neutra.

\section{REFERENCIAS}

Cabrera, J. (2016). Estudio de la corrosión de acero al carbono AISI 1020 en soluciones cloradas y su dependencia con la rugosidad del material. Tesis de doctorado en ciencias de la ingeniería. Santiago de Chile: Universidad de Chile.

Castañeda, A. (2014). Las pérdidas económicas causadas por el fenómeno de la corrosión atmosférica del acero de refuerzo embebido en el hormigón armado. Revista CENIC Ciencias Quimicas, 45, 52-59.

Foncubierta, M. (2017). Diseño de una línea de pretratamiento anódico para piezas de aluminio a termolacar bajo las directivas de la marca de calidad EWAA-EURAS. Tesis de diploma e ingeniería química. Cadiz: Universidad de Cádiz.

González, L. (2013). El aluminio lacado como alternativa segura de materiales para la construcción. Tesis de maestría, La Habana: Universidad Tecnológica José Antonio Echeverría.

Lluveras, E., Martínez, J., González, L. \& Fundora, J. (2018). Aplicación de software estadísticos y modelos matemáticos para la evaluación de la velocidad de corrosión en el acero. Revista U.D.C.A Actualidad \& Divulgación Científica, 21(1), 179-186.

Olaya, J. \& Torres, M. (2012). Resistencia a la corrosión de recubrimientos orgánicos por medio de espectroscopía de impedancia electroquímica. Ingeniería y Universidad, 16(1), 43-58.

NC ISO 2808 (2010). Pinturas y barnices- determinación del espesor de película, pp. 26-32.

NC ISO 4628-1 (2007). Pinturas y Barnices-Evaluación de la degradación de los recubrimientos de pintura. Designación de la intensidad, cantidad y tamaño de los tipos más comunes de defectos-Parte 1: Principios generales y esquemas de evaluación. pp. 1-9.

NC ISO 4628-2 (2007). Pinturas y Barnices-Evaluación de la degradación de los recubrimientos de pintura. Designación de la intensidad, cantidad y tamaño de los tipos más comunes de defectos- Parte 2: Designación del grado de ampollamiento. pp. 1-17.

NC ISO 4628-3 (2007). Pinturas y Barnices-Evaluación de la degradación de los recubrimientos de pintura. Designación de la intensidad, cantidad y tamaño de los tipos más comunes de defectos-Parte 3: Evaluación del grado de oxidación. pp. 1-18. 
NC ISO 4628-4 (2007). Pinturas y Barnices-Evaluación de la degradación de los recubrimientos de pintura. Designación de la intensidad, cantidad y tamaño de los tipos más comunes de defectos-Parte 4: Evaluación del grado de agrietamiento. pp. 1-18.

NC ISO 4628-5 (2007). Pinturas y Barnices-Evaluación de la degradación de los recubrimientos de pintura. Designación de la intensidad, cantidad y tamaño de los tipos más comunes de defectos-Parte 5: Evaluación del grado descamación. pp. 1-9.

NC ISO 4628-6 (2007). Pinturas y Barnices-Evaluación de la degradación de los recubrimientos de pintura. Designación de la intensidad, cantidad y tamańo de los tipos más comunes de defectos-Parte 4: Evaluación del grado de enyesado por el método de la cinta. pp. 1-8.

NC ISO 9227 (2014). Ensayos de corrosión en atmósferas artificiales - Ensayos en rociado salino. pp. 6-28.

UNE EN ISO 2360 (2004). Recubrimientos no conductores sobre materiales base no magnéticos. Medición del espesor de recubrimiento. Método por corrientes inducidas sensibles a la variación de amplitud. pp. 1-10. 


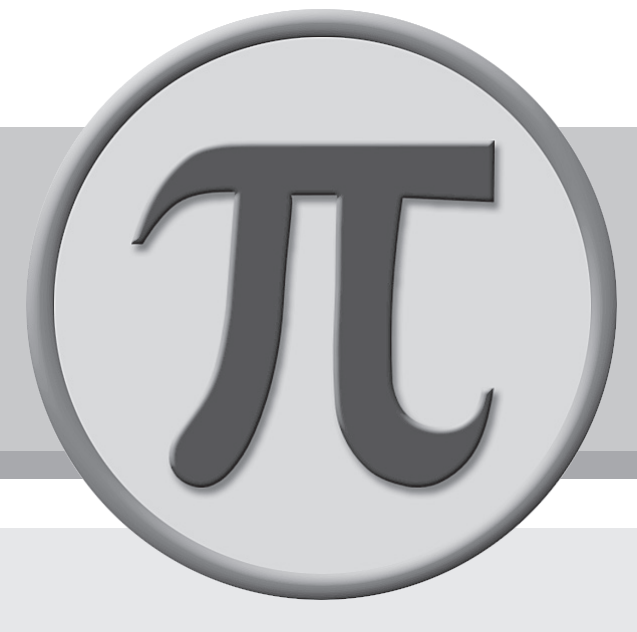


